\title{
Social Survey and Problem About Phobias Affecting People Daily Lives
}

\author{
Tianqi Wang \\ School of Rugby, Rugby, CV22 5DU, UK *Corresponding author Email: yaqing1028@qq.com
}

\begin{abstract}
In this essay, it will talk about what specifically phobia is and clearly define and differentiate it with other fear. It will also put phobia into groups and detailly explain the impact of having a phobia. With regards to that, the essay will also talk about the therapy people can take to treat or overcome the phobia. This essay also includes a public survey done by the author to suggest how faintly random civilians know about phobia and how common phobias actually are. In the conclusion it showed how unaware people these days are without acknowledging the inconvenience of people with phobia and talked about what people could invent to benefit and ease the life of people with phobia
\end{abstract}

Keywords: Phobias, anxiety disorder, scare, amygdala, hormones.

\section{INTROCTUTION}

Have you notice that more and more people around you have phobias these days? A lot of people are stressed or pressured by unnecessary objects or situations. [1] They sweat or shiver while at public spaces. These factors not only effect their life, but also other people, since the increase of phobia meant the loss of many job opportunities. For instance, people who are fear of heights can't work at high level sights or blocks; people with animal phobia can't work in a zoo; or people with agoraphobia can't work in a crowded space. Phobias are more and more common nowadays due to the pressure in life not only financially but also mentally.

\subsection{The idea}

The idea of bringing out a social survey is to check and assess people's awareness on phobia and also works in a way that increase publicity of phobia since people could not have notice or know how troubling. I heard a lot of people around my age group trying to laugh at people with phobia or anxiety order, so I became fairly interested by how phobia work and the function inside our body. Then I soon realise that people might have heard about phobia, but just not aware of what it is and how it is troublesome. Therefore, I wanted to do a public survey.

\subsubsection{Survey function}

Citations with regard to my relatives living in different regions to help with the survey. In this work, it contained answers from three region which is Sweden, China, and England. Both paper and digital record is done but majority being paper and collected data.

\subsection{Evolution of phobia}

One of the reasons why nowadays, more and more people have phobia is because they are so reliant on things such as their mobile phone, or their book. People cloud ask a random person walking on a street: what he/she could want the most when they are thrown to a deserted land. The research shows that aside from food or water, there's a higher probability that they would say mobile phone. [2] As we are so reliant on it, people nowadays are having Nomophobia which is a fear of being without their mobile phones or missing the signal on the mobile phone. Smart phones have become so ubiquitous that we are unable to remain the part of these world system activities without this access to these mobile devices. A study shows that 10 million people in UK suffer from different types of phobias, and yet researches which goes into cases and interventions are incredibly small compared with different psychological health considerations.

\subsubsection{Oringination}

An English researcher: Nicky Lidbeter, who was the 
chief head of Anxiety Britain, gets herself suffered from phobias and is one great advocate of CBT interventions, which one of the reasons is because she suffers from phobias herself as well.[3] Therefore, that is her motivation for researching phobia. How do we even get a phobia then? Genetic and environmental factors may have affected phobias. Kid who has a near relation with the anxiety disorder are at risk of developing the phobia. Disturbing events, such as almost drowning, may take on a phobia. Exposure to restricted spaces, intense heights, and creature or insect bites may all be the origins of phobia.

\section{CLASSIFING PHOBIA}

However, what is phobia? I would guess that people nowadays do not realise how phobias are affecting our life. Phobia is a form of anxiety disorder that depicts the unreasonable and irrational mood towards a single object, action, or condition. [4] Phobias require extreme emotions surrounding an object or condition that realistically poses little or no serious risk. They are distinct from general concerns in that the associated emotion is so powerful it interferes their daily life.

\subsection{Specific phobia and Complex phobia}

Phobias are split into different phobias, cultural phobia, and agoraphobia. Types of particular phobia include those to specific animals, physical environment situations, people or loss, and specific situation. The most common represent emotion of spiders, anxiety of snakes, and anxiety of heights. Occasionally they are triggered by the bad experiences with this objector place.[4] Social phobia is when this condition is feared as this individual is upset about others judging them. Agoraphobia is when emotion of the ability to work normally. People suffering from phobias may get to extreme lengths to prevent happening or feeling the dreaded object or condition. [4] With specific phobias, it is easy to avoid, and people get alone very easily with these phobias. Say for example, if you are scared of heights, you can easily avoid it by not travelling to high places and just by doing that, their life goes on as normal. However, with complex phobias, it is very hard to avoid. Therefore, it is a massive impact on their daily lives. Social phobia for instance, they need to avoid talking in groups; public speaking; speaking to new people such as the doctor or a therapist; or even speaking on a phone. As a result, people with complex are much problemed in real life than specific phobias.

\subsection{Fear (Uncanny Valley Theory)}

Moreover, how do we differentiate between real life dangers and phobias? There's a very interesting phobia on the internet these days, it is the fear of clowns. Generally, we class clowns as an object that brings us joy and happiness. However, since when did we relate clowns with a scary and uneasy figure? [5] In 1970, a robotics professor called Masahiro Mori had introduced a theory call "The Uncanny Valley Theory", the hypothesis states when a human made figure that has the appearance of a human, and with a robotic voice and responses which were highly positive and enthusiastic. [5] This at the beginning started off fine such as a doll or a talking toy. However, as these "toys" or robots gets more and more real or as close to human as possible, they make people uneasy or scared. Just imagine a human-like-robot talking in an un-human or unnatural way. [5] Which, the name "The Uncanny Valley" comes from a graph showing the happiness of the audience when the object that they are showed gets more and more alike to human. Everyone started off with great fun when they are showed a toy, then it is dropped when a talking doll is showed. Afterwards, they are showed a talking robot with a human body and acts weirdly, everyone's rate of happiness towards that object dropped massively. However, when a robot is proved to be a hundred precent human-like, everyone seems to be happy with it and that drop showed on the graph is name "The Uncanny Valley".

\subsubsection{Common fear}

Besides that, there is so many things you can fear, such as sickness. People are scared of sickness, firstly, because it makes you unwell or feel bad. It makes you tired and stressed, even worse: to die off deadly diseases such as aids or cancer. Moreover, people are also scared of losing their job, since losing their job could mean that they must find a new job. As a result, general fear means people are scared or try to avoid new things or stress. On the other hand, having a phobia means that you get stressed even though they can't avoid it. They get scared only with a specific object. That is one of the main reasons that assorts real life fear and phobias.

\section{FUNCTIONS WITHIN THE BODY}

How does phobia work in our brains or how does memory even get stored in our brains? According to lots of websites including NHS and world health care, all the memories that relates to fear goes into a part of a nuclei that is called amygdala. [6] An amygdala is a part of the nuclei that shapes like an almond that is situated in the deep-down brain's medial temporal lobe. It stores objects or situations that is related to fear, and it is bonded with phobias like social phobia or other anxiety. The function of the amygdala is to respond and reflect stimuli associated with fear.[6] Like classical condition, amygdala remembers to relate or link an object or situation to a negative or avoidant responses, creating a feeling of fear of phobic holders. In this way, the amygdala is responsible for not only recognizing certain object or situations or things as dangerous but plays a role in the 
storage of threatening things to memory.

\subsection{Hormones released}

There is also two other parts that affect phobia which is the basolateral nuclei and the hippocampus interact with the amygdala in the brain, which suggests why memories are often remembered more vividly if they have emotional or physical significance. In addition to memory, an amygdala nucleus also triggers the hormones that affect fear and irrational emotion. When the fear or aggression response is triggered, the amygdala releases hormones into the body and put the human body into an alert state, which prepares a person to be aggressive and fearful. [6] This defensive alert state and response are known as the fight-or-flight response. Which basically means the person either get very irrational or they just keep it inside themselves. Inside the brain, however, this stress response can be seen in the hypothalamic-pituitary-adrenal axis, this group works their way out in receiving the impulse that states seeing the object or situation, intercepting it and release hormones into the blood. Then the neurons of the hypothalamus release corticotropin-releasing hormone, which is sent to a part of the brain called the anterior pituitary. It releases adrenocorticotropic hormone, which ultimately stimulates the release of cortisol which is one of the mainstreams of hormone which controls a person's mood or emotion.

\subsubsection{Cortisols}

In relation to anxiety, the amygdala is responsible for activating the hypothalamic-pituitary-adrenal axis, while the hippocampus is responsible for suppressing it. Glucocorticoid receiver in the hippocampus monitors the amount of cortisol in the system and through negative feedback can tell the hypothalamus to stop releasing cortisol. [6] Therefore, that is why people with phobias clams down a lot when the object or situation is gone. Studies on mice researched these mice which had high concentrations of cortisol showed higher levels of anxiety, while those mice which had no, or low amounts of cortisol were less anxious. In people with phobias, therefore, high amounts of cortisol may be present, or alternatively, there may be low levels of glucocorticoid. That explains what hormones are released in the body when fear is present in the body of people who has phobias.

\section{PUBLIC SURVEY}

Furthermore, with regards to my research. I was aiming to find out how phobias are affecting people's life in different year groups. I have asked them 3 questions, the first questions asking if they have or knew people that has phobia. The purpose of asking this question is to know how high the percentage of people who have phobia is and to know how people feel about or think about phobic people.

\subsection{First question}

\subsubsection{Children}

Firstly, most of younger age from 7 to 12 years old children's response is that they haven't seen anyone with phobia or don't know what phobia is. However, by the time when phobia is defined to them, a lot of them said that they will be happy and willing to help these kinds of people to avoid things or situations that they fear. Which, for sure is a nice thing to say and it very vividly expressed and showed how innocent these children are. It is most likely because they don't really know how complicated and troubling having a phobia is. However, there is a small girl who said that she discovered that she has social phobia at the age of 5 and she don't have many friends until now. She says that she is trying her best to talk to other people, but it most likely it makes her weird because of the things she said. She had lots of things she could share with other people. Such as how school have been and all these years of experiencing having social phobia. This is a very good example showing how phobia could really have or have not make a child's childhood memory very catastrophic.

\subsubsection{Adults}

On the other hand, the adults responded very calmly and detailly whether they knew or not knew/ have or not have a phobia, and they will write about what is their phobia and what they try to avoid; their life with that phobia; and are they taking therapy and what indeed is the therapy. The main reasons for that are because they have been experiencing life phobia most of their life so they might even be more familiar with it then some random doctor.

\subsubsection{Old people}

Lastly, when asking elder people if they have experience phobia, most of them would say no because it is either they don't have any phobia, or it have been cured a long time ago so that they forgot the fact that they had a phobia. Despite that there is one old woman who said she had acrophobia when she is younger, but she got less aggressive when she got older. That is because the amount of cortisol released into the bloodstream decreases, therefore a person's reaction or aggressiveness gets calmer and more peaceful.

\subsection{Second question}

Moving onto the second question, I asked if the phobia they had have impacted their life. I asked this question because I wanted to find out how phobia is 
affecting people's daily life. It is for sure that people must be troubled by these difficult-dealing phobias, but how deeply and detailly it is actually impacting their life is what I am going to find out.

\subsubsection{Children}

Towards the younger children, I asked what they would imagine life would be like if they had a specific phobia. Most of their answer is that they would get very depressed or sad that they don't even want to live properly. These answers are actually worrying, because what they are mostly suggesting is that people with phobia are troubled so much, that they can't even live properly. However, this is normally not the case because they are still a high percentage in the people who has phobias that they live properly. They could always find a way to live a life avoiding meeting or seeing these object or situations. Therefore, children are still misunderstanding the concept that people with phobia can still live a normal life.

\subsubsection{Adults and Old people}

With regards to the middle-aged people, they clearly told how phobias were affecting their life. Such that one person who had claustrophobia by being trapped in the elevator when he was small. As a result, he always sweat in the elevator while going up to work. He was really troubled by it and very worry that one day he would be so depressed and finish his life youngly. It is very pathetic how a person might think like this, but it is the fact that something like this might happen to him, as he gives up trying so hard to live.

\subsection{Last question}

Finally, my final question is whether their phobia is cured and if yes, how. This question is asking for different therapies or medications different people take. Related medications are towards the fear of an object or situation.

\subsubsection{Medication}

Research shows that Antidepressant medications such as SSRIs or MAOIs are really helpful in some cases. SSRIs work as a neurotransmitter in the brain. Since the part in the brain that effects the mood are prescribed by an antidepressant, sedative such as benzodiazepines may also be prescribed, which will help people with phobia to relax and calm down. As well as that, beta blockers are also medicinal option as they could stop or reduce the effects of adrenaline, such that they stop sweating or the increase of heart rate. By taking it before a phobic event could decrease the symptoms, making people less frightened.

\subsubsection{Hypnotherapy}

Another therapy could be hypnotherapy. They fact that hypnotherapy can be used alone just made life way easier towards treating phobia. Since that the phobias will all be triggered by an event or memory, the brain will repress traumatic memories from the conscious mind until the person's body is ready to deal with it. The effect of a hypnotherapy, is that it makes the person into an extreme relax condition so that the person if more open to suggestions and desire to change. Making the memory to stop reacting fearful towards those object or situations.

\section{CONCLUSION}

In conclusion, phobias are indeed effecting people's daily life, and it is a problem spreading across the world. However, I believe that phobias will be less and less due to the development of medicine and therapy. People could be more aware of the importance of phobia since this showed show unacknowledged people are now with regards to phobia. It will definitely be a great idea to put phobia into consideration while schools are teaching health and disease in order to increase public awareness. In the future, with regards to those advancing technologies, scientists may as well discover a way in which people could selectively choose to delete parts of their memory without side effects. This would not only be a great tool in helping people with phobia, but also normal people could need this type of tool as well.

\section{REFERENCES}

[1]: NHS UK. (2018) Symptoms-phobia https://www.nhs.uk/mentalhealth/conditions/phobias/symptoms/

[2]: Wolters Kluwers UK. (2019) Nomophobia https://www.ncbi.nlm.nih.gov/pmc/articles/PMC65 10111/

[3]: Nicola Slawson UK. (2016) Nicky Lidbetter https://www.theguardian.com/society/2016/may/24 /icky-lidbetter-my-anxiety-has-been-motivatormental-health

[4]: NHS UK. (2019) Phobias https://www.nhs.uk/mentalhealth/conditions/phobias/

[5]: (2020) Uncanny Valley Theory https://en.wikipedia.org/wiki/Uncanny_valley

[6]: Ana Soudoiu UK. (2018) https://www.medicalnewstoday.com/articles/32349 2\#Triggering-the-response 